\title{
Seelsorgliche Kirche in viralen Krisen-Zeiten ... und darüber hinaus
}

\author{
Schlag, Thomas
}

\begin{abstract}
Der vorliegende Beitrag vertritt und entfaltet die These, dass seelsorgliche Praxis in der aktuellen Krise eine wichtige Orientierungsmarke darstellt, durch die aufgrund digitaler Transformationsprozesse neues Nachdenken über die seelsorgliche Präsenz von Kirche in vielfältiger Gestalt auch über die Krise hinaus notwendig ist. Von einer grundsätzlichen poimenischen (seelsorgetheorischen) Perspektive aus werden aktuelle Herausforderungen benannt sowie am Beispiel erweiterter Telefonseelsorgeangebote Einblicke in aktuelle Formen digitaler seelsorglicher Praxis in Coronazeiten gegeben. Von dort aus werden in theologischer Perspektivierung Kernaspekte seelsorglicher Praxis unter Krisenbedingungen benannt sowie Folgerungen für die Zukunft seelsorglicher Praxis im Kontext einer Kultur der Digitalität angestellt.
\end{abstract}

DOI: https://doi.org/10.1515/spircare-2020-0081

Other titles: Pastoral care and the church in times of the viral crisis ... and beyond

Posted at the Zurich Open Repository and Archive, University of Zurich

ZORA URL: https://doi.org/10.5167/uzh-189427

Journal Article

Published Version

Originally published at:

Schlag, Thomas (2020). Seelsorgliche Kirche in viralen Krisen-Zeiten ... und darüber hinaus. Spiritual Care, 9(3):265-272.

DOI: https://doi.org/10.1515/spircare-2020-0081 


\title{
Originalia
}

\author{
Thomas Schlag*
}

\section{Seelsorgliche Kirche in viralen Krisen-Zeiten ... und darüber hinaus}

\author{
Pastoral care and the church in times of the viral crisis ... and beyond
}

https://doi.org/10.1515/spircare-2020-0081

Vorab online veröffentlicht 23. Juli 2020

Zusammenfassung: Der vorliegende Beitrag vertritt und entfaltet die These, dass seelsorgliche Praxis in der aktuellen Krise eine wichtige Orientierungsmarke darstellt, durch die aufgrund digitaler Transformationsprozesse neues Nachdenken über die seelsorgliche Präsenz von Kirche in vielfältiger Gestalt auch über die Krise hinaus notwendig ist. Von einer grundsätzlichen poimenischen (seelsorgetheorischen) Perspektive aus werden aktuelle Herausforderungen benannt sowie am Beispiel erweiterter Telefonseelsorgeangebote Einblicke in aktuelle Formen digitaler seelsorglicher Praxis in Coronazeiten gegeben. Von dort aus werden in theologischer Perspektivierung Kernaspekte seelsorglicher Praxis unter Krisenbedingungen benannt sowie Folgerungen für die Zukunft seelsorglicher Praxis im Kontext einer Kultur der Digitalität angestellt.

Schlüsselwörter: Seelsorge, Digitale Kommunikation, Pastorale Präsenz, Digitale Kultur, Krise

\begin{abstract}
This paper develops the argument that the practices of pastoral care in the current crisis represent an important orientation mark, through which, due to digital transformation processes, new thinking about the pastoral presence of the church in manifold forms is necessary even beyond the crisis. From a fundamental poimenical perspective, current challenges will be identified and insights into current forms of digital pastoral practice in corona times will be given using the example of extended telephone pastoral care services. From there, core aspects of pastoral practice in this time of the crisis will be identified and conclusions for the future of pastoral practice in the context of a culture of digitality are drawn.
\end{abstract}

*Korrespondenzautor: Prof. Dr. Thomas Schlag, Theologische Fakultät - Praktische Theologie, Universität Zürich, Schweiz,

E-Mail: Thomas.schlag@theol.uzh.ch
Keywords: pastoral care, digital communication, pastoral presence, crisis, digital culture

\section{Einleitung}

Die Corona-Pandemie fällt in die Osterzeit des Jahres 2020 und reicht doch in ihren Wirkungen über die Tatsache entfallener analoger Ostergottesdienste weit hinaus. Nicht nur durch das Verbot gottesdienstlicher Versammlungen, sondern auch aufgrund der massiv eingeschränkten Kontaktmöglichkeiten hat sich aktuell in vielen Kirchengemeinden die Frage nach digitalen Kommunikationsund Interaktionsmöglichkeiten erheblich verstärkt und in gewisser Weise auch verschärft.

Was bereits seit einigen Jahren unter dem Stichwort einer „Kirche vor und in der digitalen Transformation“ vor allem im angelsächsischen Raum breit diskutiert wird (etwa Hutchings 2017), ist durch die aktuelle Krise zu einer nochmals deutlich gesteigerten Herausforderung sowohl für das pastorale Personal wie für die kirchliche Arbeit auf den unterschiedlichen Handlungsfeldern von Gottesdienst und Kasualpraxis, Seelsorge, Bildung, Diakonie, Gemeindeentwicklung und Kommunikation geworden. Die Organisierung und das Selbstverständnis kirchlicher Praxis stehen damit grundsätzlich vor erheblichen Transformationsanforderungen, die sich weit über technische Aspekte hinaus auf die Frage einer ekklesiologisch stimmigen Gesamtpräsenz in einer „Kultur der Digitalität“ (Stalder 2017) beziehen.

Nun haben sich angesichts des Zusammenfallens von Pandemie und Osterzeit tatsächlich viele der bisherigen Aktivitäten und auch die öffentliche Wahrnehmung vor allem auf das gottesdienstliche Angebot und die Frage zukünftiger Formate konzentriert, was kaum verwunderlich ist. Denn zuerst einmal ging es naturgemäß darum, das gottesdienstliche Programm unter den veränderten Bedingungen aufrechtzuerhalten bzw. in nun mögliche neue digitale Formen hin $\mathrm{zu}$ transformieren (zum Überblick Campbell 2013). 
Exemplarisch spiegelt sich diese Vielfalt auf der Internetseite der Evangelischen Kirche in Deutschland „Kirche von zu Hause - Alternativen (nicht nur) in Zeiten von Corona“ (EKD 2020) wider, die deutlich macht, dass auf vielen Handlungsfeldern gegenwärtig innovative Überlegungen zur digitalen Präsenz angestellt werden. Auch einzelne Landeskirchen weisen hier inzwischen eigene Seiten aus, so etwa die Evangelisch-Lutherische Kirche in Norddeutschland (Nordkirche 2020) oder die Evangelische Landeskirche in Württemberg (ELiW 2020). In der Schweiz kann auf das Angebot der Evangelisch-reformierten Kirche Schweiz verwiesen werden (EKS 2020). Die erste empirische Studie (MacDonald et al. 2020a; 2020b) sowie eine bereits erschienene umfangreichere akademische $\mathrm{Ab}$ handlung reflektieren diese Schwerpunktsetzung (Campbell 2020a, siehe auch zur Einschätzung eher essayistisch Campbell 2020b).

Es zeichnet sich allerdings ab, dass über die gottesdienstliche Verkündigung hinaus durch diese Krise jetzt in aller Schärfe wahrgenommen wird, dass die pastorale Praxis inmitten einer Kultur der Digitalität schon längst vor erhebliche Herausforderungen gestellt ist, die auch andere kirchliche Handlungsfelder sowie das pastorale Selbstverständnis betreffen.

Da sich die Beiträge des vorliegenden Sonderheftes insbesondere auf die Spiritual Care Praxis im Zusammenhang mit COVID-19 beziehen, sollen im Folgenden von einer grundsätzlicheren poimenischen Perspektive aus aktuelle Herausforderungen benannt (1.) sowie Einblicke in aktuelle Formen digitaler seelsorglicher Praxis in Coronazeiten (2.) gegeben werden. Von dort aus werden in theologischer Perspektivierung (3.) Kernaspekte seelsorglicher Praxis unter Krisenbedingungen benannt (4.) sowie abschließend Folgerungen für die Zukunft seelsorglicher Praxis angestellt (5.).

\section{Gegenwärtige Herausforderungen}

In poimenischem (seelsorgetheoretischem) Sinn stellt sich für sich die praktisch-theologische Reflexion wie für die kirchliche Praxis die Herausforderung, ob und in welcher Hinsicht die Qualität und Verlässlichkeit von personalen Begegnungen auch in Zeiten der primär digitalen Kommunikation und in dieser Situation der "Aktualkrise“ (Rössler: 2016, 458ff.) auf möglichst menschengemäße Weise näher konzipieren und praktisch umsetzen lassen. Mindestens im Bereich der deutschsprachigen Praktischen Theologie ist die Beschäftigung mit diesen Fragen bisher noch erstaunlich überschaubar (wesentlich hier Nord 2016; Luthe 2020; Berger-Zell 2014; Knatz 2013 und eben- falls aus jüngerer Zeit die empirisch angelegte Dissertation von Waclawiak 2014; an älteren Beiträgen sind zu nennen (Bobert 2000, Knatz \& Dodier 2003, Gehring 2002 sowie das Feld instruktiv aufmachend Nord 2009. Interessanterweise ist im weiten Feld der inzwischen intensiven „Digital Religion“-Forschung die Thematik der Seelsorge gegenüber Fragen des pastoralen Selbstverständnisses und der Gemeindebildung deutlich weniger stark bearbeitet: dazu jetzt v.a. K.A. Bingaman mit seinem Plädoyer für "contemplative-mindfulness practices of pastoral care and counseling “ (Bingaman 2018).

Damit eröffnet sich sogleich die weiterreichende pastoraltheologische Frage danach, was im Selbstverständnis von Pfarrerinnen und Pfarrern den Kern ihrer seelsorglichen Rolle gerade unter digitalen Bedingungen ausmacht. In ekklesiologischer Hinsicht kommt die weitergehende Frage hinzu, ob nicht auch die längst diskutierte Frage nach digital induzierter Partizipation auch auf dem Feld der Seelsorge einen erheblichen Schub im Sinn des Priestertums aller Gläubigen erfahren könnte und sollte. Damit stellt sich die noch weiterreichende Frage, was den tieferen theologischen Sinn einer sich seelsorglich verstehenden Kirche ausmacht und wie sich dieser in digitalen Zeiten und durch die entsprechende Kommunikationspraxis sowie im Zusammenspiel der unterschiedlichen gemeindlichen Ämter (Hermelink 2020; Hauschildt 2013) und theologieproduktiven Gestaltungsmöglichkeiten (Müller 2019) erkennbar machen lässt.

Die These der folgenden Ausführungen besteht darin, dass seelsorgliche Praxis in der aktuellen Krise als eine wichtige Orientierungsmarke verstanden werden sollte, durch die aufgrund der - jetzt nur beschleunigten digitalen Transformation - ein neues Nachdenken über die seelsorgliche Präsenz von Kirche in vielfältiger Gestalt auch über die Krise hinaus notwendig ist.

\section{Einblicke in aktuelle Formen digitaler seelsorglicher Praxis in Coronazeiten}

Eindrücklich wird schon in der aktuellen Handreichung für Seelsorger/-innen (Roser et al. 2020) deutlich, worin die gegenwärtigen krisenbedingten Herausforderungen im Bereich der Krankenhausseelsorge bestehen. Diese liegen zuallererst darin, dass die bisher gewohnte personale Nähe zu Menschen in Krisensituationen so nicht mehr ausgeübt werden kann wie dies bisher ganz selbstverständlich der Fall war. Dass dies in Situationen des Lebensendes von einschneidender Dramatik sowohl für Seelsorgende, Angehörige, das Pflegepersonal und die sterbenden Menschen selbst ist, muss kaum eigens betont werden. 
Nun liegen - über dieses spezifische Arbeitsfeld hinaus - Herausforderungen aber auch außerhalb des Krankenhauses überall dort, wo der bisherige seelsorgliche Auftrag nicht mehr in der bisher gewohnten Weise ausgeübt werden kann: Dies gilt für Geburtstagsbesuche ebenso wie für Trauergespräche, für Beratungen in Krisensituationen ebenso wie für die gemeinsame Vorbereitung von Tauf-, Konfirmations- oder Hochzeitsgottesdiensten und dies ist nicht zuletzt für die ganz alltäglichen seelsorglichen Begegnungen bei Gelegenheit festzuhalten, die in den Zeiten der Ausgangsbeschränkungen und des „,SOcial distancing" ebenfalls erheblich erschwert bzw. unmöglich geworden waren. In all diesen Fällen wurde die für die seelsorgliche Beziehung notwendige personale Begegnung "face to face" und damit die wechselseitige Wahrnehmung der Gesprächpartner/-innen in geradezu disruptiver Weise tangiert.

Die Kirchen und Kirchgemeinden versuchen inmitten der Krise durch eine ganze Reihe unterschiedlicher Angebote zu reagieren, bei denen intensiv auf unterschiedlichste digitale Techniken und Formate zurückgegriffen wird. So ist es kaum verwunderlich, dass die Telefonseelsorge gegenwärtig einen starken Boom erlebt. Auch andere Formen der signalhaften und auch ganz materialen seelsorglichen Zuwendung im Sinn neu organisierter Sorgenetze werden erprobt: Die Kontaktaufnahme zu Älteren, denen die neuen Medien nicht vertraut sind, über Telefon (das natürlich längst oft ein digitales Smartphone oder Tablet ist) und tatsächlich auch auf dem klassischen Postweg, durch die logistische Unterstützung bei Nachbarschaftshilfeprojekten, die Versendung von „Care-Paketen“ oder auch die Zustellung von Lebensmitteln nach vorübergehender Schließung der etablierten örtlichen Tafel-Strukturen.

Besonders interessant sind für den vorliegenden $\mathrm{Zu}$ sammenhang gerade solche Angebote im deutschsprachigen Kontext, die die digitalen Möglichkeiten nutzen, um die bisherigen Formen der persönlichen Begegnung wenigstens ein Stück weit zu kompensieren.

Unterschiedliche Angebote aus sehr verschiedenen Kontexten seien hier im Folgenden genannt, wobei erwähnt werden muss, dass diese Angebote zwar keineswegs erst durch die Krise selbst entstanden sind, nun aber erhebliche Dynamik zu gewinnen scheinen. Interessanterweise werden selbst im neuesten Standardüberblick zur Telefonseelsorge wie im Handbuch insgesamt, digitale Formate praktisch komplett ignoriert (Hücker 2016, hingegen schon Morgenthaler 2009:361) und damit der erhobene Anspruch auf eine adressaten- und situationsgerechte Seelsorge (vgl. etwa Bukowski 2016: 260) nicht eingeholt.
Hingewiesen sei hier auf die bereits im Jahr 2003 gegründete sogenannte Chatseelsorge als Angebot der Evangelisch-lutherischen Landeskirche Hannovers und der Evangelischen Kirche im Rheinland. Laut Selbstauskunft will dieses Angebot „für alle Menschen, unabhängig von Religionszugehörigkeit, Alter und Geschlecht, ein Seelsorge- und Beratungsangebot ausschließlich im Chat machen, das die Vertraulichkeit gewährleistet und die Anonymität des Chatpartners achtet“. Die Seelsorger/-innen und Moderator/-innen verfügen für die Angebote von Zweiergesprächen einerseits und offenen Chats andererseits über die notwendige Ausbildung in Seelsorge oder Beratung. Die aktuelle Situation wird durch einen Hinweis auf das erweiterte Angebot deutlich widergespiegelt, wenn es heißt: „Das Coronavirus unterbricht viele vertraute Strukturen. Menschen haben Angst. Menschen vereinsamen. Die Chatseelsorge hat in dieser Zeit ihre Seelsorgezeiten erweitert.“ (http://chatseelsorge.evlka.de)

Als zweites Beispiel sei die „Dargebotene Hand“ genannt, ein Zusammenschluss von zwölf lokal und regional verankerten, unabhängigen Organisationen unter einem gesamtschweizerischen Dachverband. Möglich sind hier sowohl Kontaktaufnahmen durch Mail wie auch Chatkontakte. Auch hier findet sich die gegenwärtige Krise gespiegelt, insofern man auf der entsprechenden Homepage mitteilt, dass „für Corona-Ängste und andere Sorgen“ die Kapazitäten erhöht werden, nachdem bereits im März 2020 laut Selbstauskunft die Gespräche um 7.5\% zugenommen haben. Die „Dargebotene Hand“ hat daraufhin ab April 2020 die Kapazitäten deutlich erhöht (https://www.143. $\mathrm{ch} /)$.

Auch ein konkretes Angebot in Österreich agiert in diesen Zeiten stärker über Chat- und E-Mail-Formen. So beschreibt die Telefonseelsorge der Diözese Linz als ihr Ziel „ein offenes Ohr, um Menschen in Krisenzeiten das Durchhalten zu erleichtern und ihnen beratend zur Seite zu stehen.“ Zugleich hält eine der Verantwortlichen fest: „Doch nicht jeder will telefonieren, gerade bei heiklen Angelegenheiten bevorzugen oftmals Jüngere Trost und Hilfe anonym via Chat oder E-Mail“ (Bauer 2020). So wurden in diesem Angebotsbeispiel mit Beginn der Corona-Krise die Sofortchats massiv ausgebaut, die sich, so die Auskunft, in dieser Zeit vervierfacht haben. Als hauptsächlich angesprochene Themen werden psychische Erkrankungen, Einsamkeit und quälende Suizidgedanken genannt: Zudem melden sich offenbar viele überforderte Eltern, vor allem Frauen, die in der Krise zuhause arbeiten und gleichzeitig die Erziehung ihrer Kinder zu bewältigen haben.

$\mathrm{Zu}$ erwähnen ist schon an dieser Stelle in ekklesiologischer Perspektive, dass in diesen digitalen Angebotsformen der Kontaktaufnahme nicht nur hauptamtliche 
Seelsorger/-innen, sondern eine erhebliche Zahl von Ehrenamtlichen und auch vermeintlich „kirchlich Distanzierten" engagiert ist: Damit zeigen sich erhebliche religionsbezogene Kompetenzen weit über das professionelle pastorale Profil hinaus (vgl. am Beispiel von facebookKommunikation und des politischen Engagements Nord 2016: 164; grundsätzlich Merle 2019).

Als ein weiteres Phänomen, auf das an dieser Stelle nur andeutungsweise eingegangen werden soll, ist hier auf die inzwischen im sogenannten evangelischen Content-Netzwerk „Yeet“ versammelten Sinnfluencer/-innen $\mathrm{zu}$ verweisen (EKD 2020). Wirft man einen Blick auf die aktuellen Instagram-, Twitter- und YouTube-Kommunikationen der hier agierenden Personen, so sind hier teilweise intensive seelsorgerliche Dynamiken zu konstatieren. Besonders prominent ist die von „Jana glaubt“ öffentlich gemachte eigene Quarantäne und COVID-19-Infektion, die erhebliche Reaktionen von Stärkung, Trost und Gebet auf Seiten der Follower ausgelöst hat. Zum anderen ergeben sich von Seiten der inzwischen mehr als 19.000 Follower von „seligkeitsdinge“ immer wieder gerade in diesen Zeiten intensive Seelsorgesituationen (so die diesen Instagram-Account betreibende Pfarrerin der Nordkirche Josephine Teske im persönlichen Gespräch mit dem Verfasser dieses Beitrags am 7. Mai 2020).

Interessant ist, dass zu diesen in der Regel überregionalen Angebotsstrukturen nun verstärkt dezidiert gemeindliche Angebote treten. In Zeiten ausfallender personal-lokaler Seelsorge verlagern sich Bedürfnisdynamiken nicht einfach auf die Nutzung überregionaler Angebote, sondern es entsteht offenbar das Bedürfnis nach verlässlicher lokaler Erreichbarkeit und Ansprechbarkeit.

Dies zeigt sich daran, dass in der Krise Pfarrerinnen und Pfarrer aus naheliegenden Gründen verstärkt auch auf digitale Korrespondenzwege setzen, seien es nun Videos, Mails und offenbar im Einzelfall auch bestimmte Chatforen. Dies wird aber auch in punktuellen Rückmeldungen von einzelnen Pfarrpersonen deutlich, wonach etwa für krisenhafte häusliche Situationen bewusst genau vor Ort Rat gesucht wird. Schon die überblickshafte Recherche führt rasch eine Vielzahl von Berichten vor Augen, in denen Pfarrerinnen und Pfarrer über deutlich gestiegene seelsorgliche Anforderungen in der Corona-Zeit berichten (exemplarisch dazu ein Artikel in der Berner Zeitung, Graf 2020 online). Das Bedürfnis nach Trost benötigt, um nochmals an die gegenwärtigen Anforderungen für die Krankenhausseelsorge zu erinnern, eben nicht nur den medizinischen Schutzanzug, sondern auch nach wie vor den geschützten lokalen Raum pastoraler Präsenz.
Damit kommen unter den gegenwärtigen, gegebenen Umständen ganz neue Formen der Seelsorge, der „Selbstsorge-Ressourcen der Gemeinde“ (Nord 2016: 168) mit erheblichen ekklesiologischen Folgewirkungen ins Spiel. Denn eines scheint deutlich: Dass digitale seelsorgliche Kommunikationspraxis auch zukünftig und nach der Krise verstärkt gefragt sein wird, wird jetzt schon klar. Jedenfalls dürfte die Kritik an den Auftritten der Instagram-aktiven Pfarrerin J. Teske durch einen Pfarrkollegen mit dem Satz „Wir sind doch schon überall“, längst von der Wirklichkeit digitaler Kultur überholt worden sein. Diese gegenwärtigen „Notfallmaßnahmen“ werfen insofern viel weitergehende Fragen auf. Denn es wäre so kurzschlüssig wie auch wenig strategisch gedacht, würde man die nun notwendig gewordenen Angebote lediglich auf diese Krisenzeit hin für relevant erachten.

\section{Kernaspekte seelsorglicher Praxis unter Krisenbedingungen}

Die bereits erwähnte Handreichung (Roser et al. 2020) benennt Kernaspekte seelsorglicher Praxis, die sich auch über die Krise hinaus auf die Wirklichkeit kirchengemeindlichen und pastoralen Handelns transferieren lassen: So wird deutlich, dass Menschen in solchen krisenhaften Situationen zuerst einmal - fast lapidar - nicht alleine gelassen werden sollen. Zum zweiten wird erkennbar, dass Trost, Annahme und Zuneigung bis hin zur körperlichen Berührung wesentliche Elemente existenzieller Begleitung sind. Schließlich wird erkennbar, dass seelsorgliche Praxis im Krisenfall mit einem oftmals äußerst fragilen System von Menschen zu tun hat, die allesamt ebenfalls auf Trost, Annahme und Zuneigung angewiesen sind.

Welche Herausforderungen bringt dies für Formen digitaler Kommunikation und Interaktion in einem weiteren Horizont mit sich? Zur Annäherung an diese Frage ist kurz darauf $\mathrm{zu}$ sprechen $\mathrm{zu}$ kommen, was $\mathrm{zu}$ den möglichen Wirkungen digitaler religiöser seelsorgebezogener Kommunikation gesagt werden kann:

In vielen Studien im Kontext der Forschungen der „Digital Religion“ wird deutlich, dass Phänomene wie Interaktion, Resonanz, Austausch und Begegnung auch in digitalen Formaten erlebt werden können (zum Überblick neben Campbell 2013 auch Cheong et al. 2012). Auf audiovisueller Ebene ist tatsächlich eine intensive Interaktionspraxis möglich.

In der erwähnten Handreichung wird als eine mögliche Form der Kontakt von Kranken mit ihren Angehörigen über Tablets erwähnt - auch hier sind Bild und Ton wich- 
tige Ressourcen für die wechselseitige Wahrnehmung und Stärkung der Gesprächspartner/-innen im jeweiligen Moment und hoffentlich auch darüber hinaus. Formen der „Telechaplaincy“ bieten Möglichkeiten der Kontaktaufnahme, die höchst bedeutsam sind. In den letzten Wochen waren hier immer wieder Bilder zu sehen, in denen Corona-Kranke etwa über das Tablet im Kontakt mit ihren Angehörigen bleiben konnten und sozusagen digital induzierte familiale Netzwerkvergewisserungen ermöglicht wurden. In diesem Sinn können solche digitalen Formen fraglos als „Resonanzkörper in Form von Bildern, Texten, Lebensgeschichten, Symbolen und Musik“ (Berger-Zell 2014: 371) angesehen werden.

Auf der anderen Seite zeigen sich insbesondere bei der Frage des hier geschaffenen Raums und des jeweiligen Raumsettings der Begegnung bestimmte Limitierungen. Diese betreffen zum einen das Medium selbst, aufgrund dessen eine Art künstlicher, wenn auch auf eigene Art „realer“ Raum geschaffen wird. Zugleich sind in diesen digitalen Begegnungen die realen Raumerfahrungen der Interagierenden in der Regel begrenzt - das äußere Setting ist nicht bedeutungslos, und so können diese Räume in vielen Fällen nicht als ein gemeinsamer Erfahrungsraum mit allen Sinnen miteinander geteilt und erfahren werden - aufgrund der hier dramatischen medizinischen Situation sowieso, die diese Erfahrung in der Regel auf wenige punktuelle Momente begrenzt.

Wenn nun gilt, dass die seelsorgliche Begegnung in erheblichem und praktisch unhintergehbarem Maß von der unmittelbaren ganzheitlichen Begegnung geprägt ist und das Erleben mit allen Sinnen über audiovisuelle Eindrücke hinausgeht, sind hier vermutlich Grenzen gelingender Kommunikation und Interaktion gegeben. Tatsächlich ist bisher weitgehend unklar, welche Wirkungen eine digitale seelsorgliche Praxis im Vergleich zur klassisch analogen Praxis erzeugen kann und ob sie nicht Defizite aufweist, die sich auch durch noch so attraktive technische Programme nicht kompensieren lassen. Hier werden durch entsprechende Forschungen im Bereich digitaler Religionspraxis in den kommenden Jahren sicherlich genauere Einsichten gewonnen werden können.

\section{Theologische Perspektiven}

Für die Einschätzung und Beurteilung einer sach- und menschengemäßen Seelsorgepraxis im Kontext digitaler Kultur sei an dieser Stelle bewusst eine theologische Perspektive eingezogen. Die erwähnte Handreichung geht bis auf wenige Andeutungen nicht explizit auf die möglichen theologischen Referenzen für die seelsorgliche Praxis am Lebensende ein.

Es ist für die Frage einer seelsorglichen Kirche in digitalen Zeiten so hilfreich wie notwendig, sich möglicher theologischer Referenzgrößen und Ressourcen zu versichern: Erinnert sei daran, dass nach biblischer Überlieferung seelsorgliche Annahme und Zuneigung mit unmittelbarer körperlicher Gegenwart verbunden sind. Diese umfassen körpersprachliche Signale des Hörens und Sehens - des Augenkontakts und des annehmenden Wortes, etwa in Form von Gespräch oder Gebet, aber auch Berührung und Umarmung. Oftmals ist - man denke an Hiob, Psalm-Worte oder neutestamentliche Heilungsgeschichten - ein Krisensetting gegeben, das durch Krankheit, Angst, Todesbedrohung, oder Exklusion gekennzeichnet ist.

Biblisch gesehen leben gelingende Begegnungen davon, dass ein realer Raum von den Gesprächspartnern bzw. -partnerinnen tatsächlich geteilt wird, was von erheblicher theologischer Bedeutung ist - seien dies häusliche oder öffentliche, eher „urbane“ oder wüstenähnliche, seien es „einsame“ oder stark bevölkerte, tägliche oder nächtliche Räume.

Zudem leben die biblisch geschilderten Begegnungen häufig von der Eröffnung und Ermöglichung eines Weges in eine Zukunft, auf dem diese existentielle Lebensbedrohung „aufgefangen“ oder sogar „aufgehoben“ ist. Man kann insofern hier von je individuellen personal-leiblichen und räumlichen Resonanzerfahrungen sprechen, die davon leben, dass sie über audiovisuelle Ausdrücke hinaus $\mathrm{zu}$ einer spürbaren Begegnung und gemeinsamen Wegmarkierungen zwischen den Interagierenden führen.

Damit stellt sich umso dringlicher die Frage, ob diese Kerngehalte seelsorglicher Aufmerksamkeit und Zuwendung auch im Modus digitaler Kommunikation gepflegt werden können.

\section{Poimenische, pastoraltheologische und ekklesiologische Folgerungen}

Geht man schon von den genannten biblisch begründeten poimenischen (seelsorgetheoretischen) Grundperspektiven aus, dann liefert dies wichtige kriteriologische Aspekte für die Frage gelingender seelsorglicher Begegnungen im digitalen Raum. Dazu ist es hilfreich, diese poimenische Dimension wie schon anfangs erwähnt, mit pastoraltheologischen und ekklesiologischen, genauer gemeindebildenden Dimensionen zu verbinden.

An dieser Stelle kann nur angedeutet werden, dass gerade die digitalen Herausforderungen das pastorale Selbstverständnis und Portfolio grundsätzlich ausweiten. 
Nicht ohne Grund finden sich nähere Beschreibungen einer „digital ministry“ im Sinn einer „digital literacy“ und Forderungen nach „digitally literate twenty-first-century ministers“. Diese sollen aber weit mehr als nur - „savvy users of the tools“ sein, sondern vielmehr als „digital missioners“ „authenticity and language proficiency“ ausstrahlen und sich als „culturally conversant“ erweisen. Als pastorale Rolle wird für die Entwicklung ,from static knowledge keepers towards a new role as dynamic conversation partners" plädiert und die Rede ist darüber hinausgehend vom Profil einer „network/networked ministry“ sowie einer „integrated practice of networked ministry“ (so die Charakterisierungen in Oliver \& Kimball 2019).

Von diesen netzwerkbezogenen Bestimmungen aus wäre es angesichts der oben angedeuteten ehrenamtlich verankerten Aktivitäten im Bereich der digitalen Telefonseelsorge kurzschlüssig, die zukünftigen Herausforderungen nur auf das pastorale Personal zu fokussieren. Denn Seelsorge im lokalen Nahraum heißt eben immer auch: Seelsorge durch lokal präsente Personen, die in ihrer Praxis eine wesentliche Gestaltungsform des Priestertums aller Gläubigen zum Ausdruck bringen.

Hier lassen sich eine Reihe von aktuellen Initiativen in der Krisenzeit durchaus so lesen, dass seelsorgliches Engagement zukünftig viel stärker im Sinn eines theologisch produktivitätsoffenen, kybernetisch relevanten Empowerments zu profilieren ist.

Dass sich damit angesichts der oftmals viel ausgewieseneren „digital literacy“ auf Seiten von Nicht-Hauptamtlichen die bisherigen Autonomie- und Autoritätsverhältnisse sowie die theologieproduktiven Praktiken im kirchengemeindlichen Kontext erheblich zugunsten der nichtpastoralen Nutzerinnen und Nutzer verschieben dürften, ist nicht nur in diesen Krisenzeiten kaum zu bestreiten.

Für eine seelsorgliche Kirche in digitalen Zeiten bedeutet dies in der Konsequenz, dass sie sich stärker als bisher mit den vorhandenen technischen Möglichkeiten und Phänomenen „virtueller Leibkörper“ (Nord 2016) auseinandersetzen muss, um genau daran die entsprechenden theologischen Kriterien zu prüfen und gegebenenfalls auch kritische Einschätzungen dieser digitalen Formate und der dadurch mediierten Praxis vorzunehmen. So sollte einerseits die mögliche positive Wirkung solcher Begegnungen im Sinn von Räumen konkreter Freiheitserfahrung (Nord 2009: 359f.) erst einmal nicht unterschätzt oder gar in Frage gestellt werden. Denn auch für digitale Kommunikation gilt: „In krisen- und konflikthaften Situationen sind Menschen berührt von dem, was mit ihnen geschieht. Sie suchen den Dialog mit dem anderen und teilen ihre Verletzbarkeit mit“ (Rössler 2016: 474).
Auf der anderen Seite steht zu befürchten, dass der reine digitale Kontakt bestimmte Reduktionismen mit sich bringt, die auch durch die noch so ausgefeilten Techniken - jedenfalls bisher - nicht kompensiert werden können. Dabei sind die datenschutzrechtlichen Probleme vermutlich noch von vergleichsweise gut lösbarer Art (Wenzel 2003; Vauseweh 2007). So ist kritisch zu bedenken, dass die Anonymität und die Sicherstellung des Beichtgeheimnisses etwa bei Nutzung bestimmter Online-Plattformen oder auch digitaler Videokonferenz-Formate bisher keineswegs rechtlich eindeutig geregelt oder gar gewährleistet ist. Hier haben die Kirchen fraglos einen erheblichen Nachholbedarf im Blick auf die Sicherstellung seelsorglicher Privatheit und Anonymität.

In jedem Fall sind aufgrund der digitalen Möglichkeitsräume (Nord 2009: 364) auch die Räume realer Begegnungskultur „offline“ neu auszuloten und zu erweitern insofern sind aktuelle Überlegungen zu einem „Blended Counseling" (Luthe 2020) ausgesprochen anregungsreich. Denn soweit man es aus bisherigen Studien zur digitalen Gemeindebildung und -vernetzung sagen kann, sind personale und symbolisierend-rituelle Begegnungen von wesentlicher Bedeutung für die Stärkung sowohl der Individuen wie auch des jeweiligen gemeinschaftlichen Netzwerkes. Am Beispiel des Tablets im Krankenhaus kann man davon sprechen, dass die Relevanz digitaler Möglichkeiten von einer schon bestehenden und wieder erhofften realen Begegnung und damit per se von einer Mischung aus Offline- und Online-Kultur lebt. In professions- und kommunikationstheoretischer Hinsicht ist festzuhalten, dass Internetseelsorge keine „Einbahn-Kommunikation“ bleiben darf. Erst unter dieser Haltungs-Voraussetzung (Waclawiak 2015: 345) vermag sich - pastoraltheologisch und kybernetisch gesprochen - die Beziehung zwischen den Akteuren und Akteurinnen in kirchlich zusammenstimmender Leitung und eben auch zwischen Seelsorger/-in und Klient/-in aufgrund sehr unterschiedlicher digitaler Kompetenz überhaupt neu zu justieren.

Finden diese Aspekte in den zukünftigen Ausgestaltungen von Kirche und kirchlicher Praxis auf den verschiedenen Ebenen verstärkte Aufmerksamkeit - und übrigens auch Anerkennung! -, dann könnte die gegenwärtige Krise im Rückblick als wesentlicher Entwicklungsschritt zu einer viel professioneller digital agierenden, partizipatorisch integrierenden und lebensrelevanten seelsorglichen Kirche wahrgenommen und verstanden werden.

Author contributions: The author has accepted responsibility for the entire content of this submitted manuscript and approved submission. 
Research funding: None declared.

Competing interests: Author states no potential conflict of interest.

Informed consent: Not applicable.

Ethical approval: The conducted research is not related to human use.

\section{Literatur}

Bauer D (2020) Online-Seelsorge in Corona-Zeiten (27.04.2020) (online) (Zitierdatum 05.05.2020), abrufbar unter https://www. nachrichten.at/meine-heimat/landsleute/doris-bauer-onlineseelsorge-in-corona-zeiten;art209551,3253662

Berger-Zell C (2014) Trauerleibsorge in Social Media. In: Nord I \& Luthe S. (Hg.) Social Media, christliche Religiosität und Kirche. Studien zur Praktischen Theologie mit religionspädagogischem Schwerpunkt. Jena: Garamond. 363-376.

Bingaman K A (2018) Pastoral and spiritual care in a digital age. The future is now. Lanham, MD: Lexington.

Bobert S (2000) Trägt das Netz? Seelsorge unter den Bedingungen des Internet. Pastoraltheologie 89:249-262.

Bukowski P (2016) Die christliche Tradition im Blickpunkt der Seelsorge. In: Engemann W (Hg.) Handbuch der Seelsorge. Grundlagen und Profile. Leipzig: Evangelische Verlagsanstalt. 259-275.

Campbell H (2013) Digital Religion. Understanding religious practice in new media worlds. Abingdon \& New York: Routledge.

Campbell H (2020a) The distanced church: Reflections on doing church online (online) Digital Religion Publications. (Zitierdatum: 1.5.2020), abrufbar unter http://hdl.handle.net/1969.1/ 187891

Campbell H (2020b) How to build community while worshipping online. A Texas A\&M professor discusses how the pandemic is changing the way people worship. The Conversation 16th of April 2020 (Zitierdatum 5.5.2020), abrufbar unter https://today. tamu.edu/2020/04/16/how-to-build-community-while-worship ping-online/

Cheong P H, Fischer-Nielsen P, Gelfgern S, Ess C (Ed.) (2012) Digital religion, social media and culture. Perspectives, practices and futures. New York: Peter Lang.

Evangelische Landeskirche in Württemberg (2020) Gemeindeleben Online. Glaube in Zeiten der Corona-Pandemie. (Zitierdatum 05.05.2020), abrufbar unter https://www.elk-wue.de/gemeinde leben-online

EKS Evangelisch reformierte Kirche Schweiz (2020) Praktische Unterstützung in der Corona-Krise (Zitierdatum 05.05.2020), abrufbar unter https://www.evref.ch/themen/coronavirus/praktischeunterstuetzung-fuer-jung-und-alt/

EKD Evangelische Kirche in Deutschland (2020) Kirche von zu Hause - Alternativen (nicht nur) in Zeiten von Corona. (Zitierdatum 05.05.2020), abrufbar unter https://www.ekd.de/kirchevon-zu-hause-53952.htm

EKD Evangelische Kirche in Deutschland (2020) yeet, das evangelische Content-Netzwerk ist gestartet. Gemeinschaftswerk für evangelische Publizistik vernetzt und unterstützt Sinnfluencer (Zitierdatum 05.05.2020), abrufbar unter https://www.ekd.de/ yeet-evangelisches-content-netzwerk-startet-53357.htm
Gehring H-U (2002) Seelsorge in der Mediengesellschaft. Theologische Aspekte medialer Praxis. Neukirchen-Vluyn: Neukirchener

Graf S (2020) Kirche in Zeiten von Corona. Jetzt hat Seelsorge ihre große Zeit (08.5.2020 online) (Zitierdatum 05.05.2020), abrufbar unter https://www.bernerzeitung.ch/jetzt-hat-seel sorge-ihre-grosse-zeit-908393010453)

Hauschildt E (2013) Allgemeines Priestertum und ordiniertes Amt, Ehrenamtliche und Berufstätige. Ein Vorschlag zur Strukturierung verwickelter Debatten. Pastoraltheologie 102: 388-407.

Hermelink J (2020) Von der theologischen zur kybernetischen Kompetenz. Aufgaben und Qualifikationen der Pfarrerin, des Pfarrers im Gefüge kirchlicher Akteure. In: Schröder B (Hg.) Pfarrer oder Pfarrerin werden und sein. Herausforderungen für Beruf und theologische Bildung in Studium, Vikariat und Fortbildung. Leipzig: Evangelische Verlagsanstalt. 445-464.

Hücker F-J (2016) Telefonseelsorge. In: Engemann W (Hg.) Handbuch der Seelsorge. Grundlagen und Profile. Leipzig: Evangelische Verlagsanstalt. 573-590

Hutchings T (2017) Creating church online: Ritual, community and new media. New York: Routledge.

Knatz B (2013) Handbuch Internetseelsorge: Grundlagen - Formen Praxis. Gütersloh: Gütersloher Verlagshaus.

Knatz B, Dodier B (2003) Hilfe aus dem Netz. Theorie und Praxis der Beratung per E-Mail. Stuttgart: Klett-Cotta.

Luthe S (2020) Seelsorge unter den Bedingungen der Mediatisierung der Lebenswelten. In: Nord I, Merle K (Hg.) Mediatisierung religiöser Kultur. Praktisch-theologische Standortbestimmungen im interdisziplinären Kontext. Leipzig: Evangelische Verlagsanstalt [ahead of print].

MacDonald A, Stetzer E, Wilson T (2020a) How church leaders are responding to the challenges of COVID-19. COVID-19 Church Survey Summary Report March 27, 2020 (Zitierdatum 1.5.2020), abrufbar unter https://exponential.org/resource-ebooks/ response-to-covid-19/

MacDonald A, Stetzer E, Wilson T (2020b) How church leaders are responding to the challenges of COVID-19. COVID-19 Church Survey Second Round Report (Zitierdatum: 5.5.2020), abrufbar unter https://exponential.org/resource-ebooks/covid-report-2/

Merle K (2019) Religion in der Öffentlichkeit. Digitalisierung als Herausforderung für kirchliche Kommunikationskulturen. Berlin: De Gruyter.

Morgenthaler C (2009) Seelsorge. Gütersloh: Gütersloher Verlagshaus.

Müller S (2019) Gelebte Theologie. Impulse für eine Pastoraltheologie des Empowerments. Zürich: Theologischer Verlag Zürich.

Nord I (2009) Die virtuelle Dimension der Seelsorge. Wege zum Menschen 61:353-366.

Nord I (2016) Seelsorge in sozialen Medien. In: Kunz R (Hg.) Seelsorge. Grundlagen - Handlungsfelder - Dimensionen. Göttingen: Vandenhoeck \& Ruprecht. 159-173.

Nordkirche. Evangelisch-Lutherische Kirche in Norddeutschland (2020) (Zitierdatum 05.05.2020), abrufbar unter https://www. nordkirche.de/glaube/digitale-kirche/

Oliver K \& Lisa Kimball (2019) Digital media for ministry: key concepts and core convictions. In: Percy M (Ed.) The Study of Ministry. A comprehensive survey of theory and best practice. London: spck. 217-232. 
Rössler B (2016) Krise, Trauma und Konflikt als Ausgangspunkte der Seelsorge. In: Engemann W (Hg.) Handbuch der Seelsorge. Grundlagen und Profile. Leipzig: Evangelische Verlagsanstalt. 451-475.

Roser T, Peng-Keller S, Kammerer T, Karle I, Lammer K, Frick E, Winiger F (2020) Die Corona-Pandemie als Herausforderung für Spiritual Care: Handreichung für Seelsorger/-innen. Spiritual Care 9:213-216.

Stalder F (2017) Kultur der Digitalität. Berlin: Suhrkamp.

Vauseweh R (2007) Onlineseelsorge. Zur Präsentation von Seelsorgeund Beratungsangeboten im World Wide Web. Erlangen: Christliche Publizistik.

Waclawiak G (2014) Gott im Netz. Religiöse Kommunikation im Internet. Fallstudien zur Internetseelsorge. Münster: Lit.

Wenzel J (2003) Vertraulichkeit und Anonymität im Internet. Problematik von Datensicherheit und Datenschutz mit Lösungsansätzen. In: Etzersdorfer E, Fiedler G, Witte M (Hg.) Neue Medien und Suizidalität - Gefahren und Interventionsmöglichkeiten. Göttingen: Vandenhoeck \& Ruprecht. 56-70.

\section{Biografische Angaben}

\section{Thomas Schlag}

Thomas Schlag, geboren 1965, ist seit 2011 Ordentlicher Professor für Praktische Theologie (Religionspädagogik, Kirchentheorie und Pastoraltheologie) an der Theologischen Fakultät und Leiter des Zentrums für Kirchenentwicklung (ZKE) der Universität Zürich in der Schweiz. Nach dem Studium der Theologie und Politikwissenschaft in Tübingen und München promovierte er in Ethik und habilitierte sich auf dem Gebiet der Religions- und Politikdidaktik. Außerdem war er von 1996 bis 2004 Pfarrer in der Evangelischen Landeskirche in Württemberg und Studienleiter an der Evangelischen Akademie Bad Boll im Bereich Jugend, Gesellschaft und Bildung. Derzeit leitet er eine interdisziplinäre Forschungsgruppe „Digitale Religion(en)“ an der Universität Zürich. Damit verbunden sind seine weiteren Forschungsfelder: Digitalisierung und theologische Kommunikationspraxis; Perspektiven der „Öffentlichen Theologie“ und „Öffentlichen Kirche“ zwischen Kirche, Staat und Zivilgesellschaft sowie Analysen und Interpretationen der religionspädagogischen Praxis in Schule und Kirche. 